\title{
Medicine Manufacturing Supply Chain Management System Using Blockchain
}

\author{
Akshada Shelke ${ }^{1}$, Aishwarya Deshmane ${ }^{2}$, Najneen Shaikh ${ }^{3}$, Suheb Shaikh $^{4}$, Gyankamal Chhajed $^{5}$ \\ Student, VPKBIET, Baramati ${ }^{1,2,3,4}$ \\ Assistant Professor, VPKBIET, Baramati ${ }^{5}$
}

\begin{abstract}
Counterfeit of medicine is major problem for consumer. Though there are more conventional methods for identifying counterfeit of medicine but they lack in security so to overcome this problem we are proposing secure software for medicine manufacturing supply chain management system by using blockchain technology. Blockchain technology is specially used in bitcoin cryptocurrency. It is characterized as an open-source, decentralized, distributed database for storing medicine information. Instead of relying on centralized intermediaries, this technology allows two parties to transact directly using duplicate, linked ledger called blockchain. Supply chain management is collection of activities required to plan, control and execute a products flow, from collecting raw materials and production through distribution to the final customer, in the most smooth and cost effective way possible. Supply Chain is beneficial when consumer or the organizations who want the information about medicine manufacturing at every stage to compare its quality. Confidential information related to medicine manufacturing is not to be unveil is accessible to authorized users.
\end{abstract}

Keywords: Counterfeit, Blockchain, Supplychain, Smart Contract

\section{INTRODUCTION}

The pharmaceutical industry has been struggling with the challenges of counterfeit medicine and the management of its supply chain for a long time. The pharmaceutical industry and governments are also worried about the public health issue. According to a BBC report,the World Health Organization (WHO) estimates that 1,20,000 people die in Africa every year from counterfeit malaria medicine alone. So, government and drug manufacturers want to ensure that everyone in the supply chain has an effective way to verify the authenticity of the medicine sold. The traditional healthcare supply chain is limited to pharmaceutical companies, hospitals, and patients. So, here we are implementing supplychain of medicine by using blockchain to avoid counterfeit problem.

Blockchain increases the efficiency and transparency of supply chains and positively impact everything from warehousing to delivery to payment. The very important things that are necessary for reliability and integrity in a supply chain are provided by blockchain. Supply chain is a system of organizations,people, activities,information and resources involved in moving a product or service from producer to consumer. Supply chain activities involve the transformation of natural resources, raw materials and components into a finish product that is delivered to the end customer. Hence, by using blockchain technology with supply chain is used to avoid counterfeit of medicines. It gives ensurity of genuine medicine to customer.

\section{LITERATURE SURVEY}

The key stakeholders in supply chain include multiple government agencies, hospitals, clinics, drug manufacturers, drug distributors, pharmacy chains, retailers. A typical pharmaceutical supply chain after a drug launch is depicted in paper.[1] The area of benefits of supply chain management with quicker customer response and fulfillment ,also with shorter lead time. Main issues related to pharmaceutical supply chain which include issues corresponding to counterfeiting, transportation issues caused by mishandling, use of improper shipping mode.[2]

The problem of counterfeit products, especially pharmaceutical products, has plagued the international community for many decades. The battle against counterfeiting remains a significant challenge. This is of significant concern, as counterfeit pharmaceutical products can cause critical illnesses and even death.[4] With the challenges and future requirements within the pharmaceutical industry for tracing products, blockchain offers the most effective and relevant solution for sharing the data securely and efficiently throughout the supply chain and product life cycle. To track and trace each product at a package level is highly difficult with technology available today.[5] Finding the substances which are mixed to make a medicine and which medicine manufactured, sold, offered for sale. Different strategies are available to achieve these goals through different combinations of public and private involvement in the pharmaceutical management cycle.[6][7]

The government agencies across the globe want to stop the public health crisis due to the counterfeiting. It can help to prevent counterfeit medicines by adding better traceability and accountability to the supply chain management of medicines.[8] Several layers of stakeholders, including suppliers, distributors, and customers, significantly increase the 
Vol. 8, Issue 5, May 2019

supply chain risk.[10] Smart contracts have gained recently attention, especially in the context of the blockchain technology. A smart contract is a contract that can verify its correctness and impose predefined rules, thus, smart contracts are self-executing and self-enforcing. Blockverify is an US company trying to introduce Blockchain into the supply chain to avoid counterfeit and forgeries.[3]

\section{III.PROPOSED WORK}

\section{Problem Statement}

The pharmaceutical industry has been struggling with the challenges of counterfeit medicine and the management of its supply chain for a long time. The pharmaceutical industry and governments are also worried about the public health issue. So, government and drug manufacturers want to ensure that everyone in the supply chain has an effective way to verify the authenticity of the medicine sold. Blockchain technology is provide a potential solution to the problems of preventing counterfeit by adding better traceability and accountability to the supply chain management of pharmaceutical products.

\section{Scope and Objective}

In this, we are implementing supply chain management to transfer medicine from manufacturer to customer by using blockchain technology. In this process customer always safeguard about the medicine which are ordered from particular manufacturer. The main aim to avoid major problem of counterfeit of medicine. Also to meet consumer demand for delivery of high quality with minimal lead time. Minimizing manpower and cost to transfer medicine from producer to consumer.

\section{System Architecture}

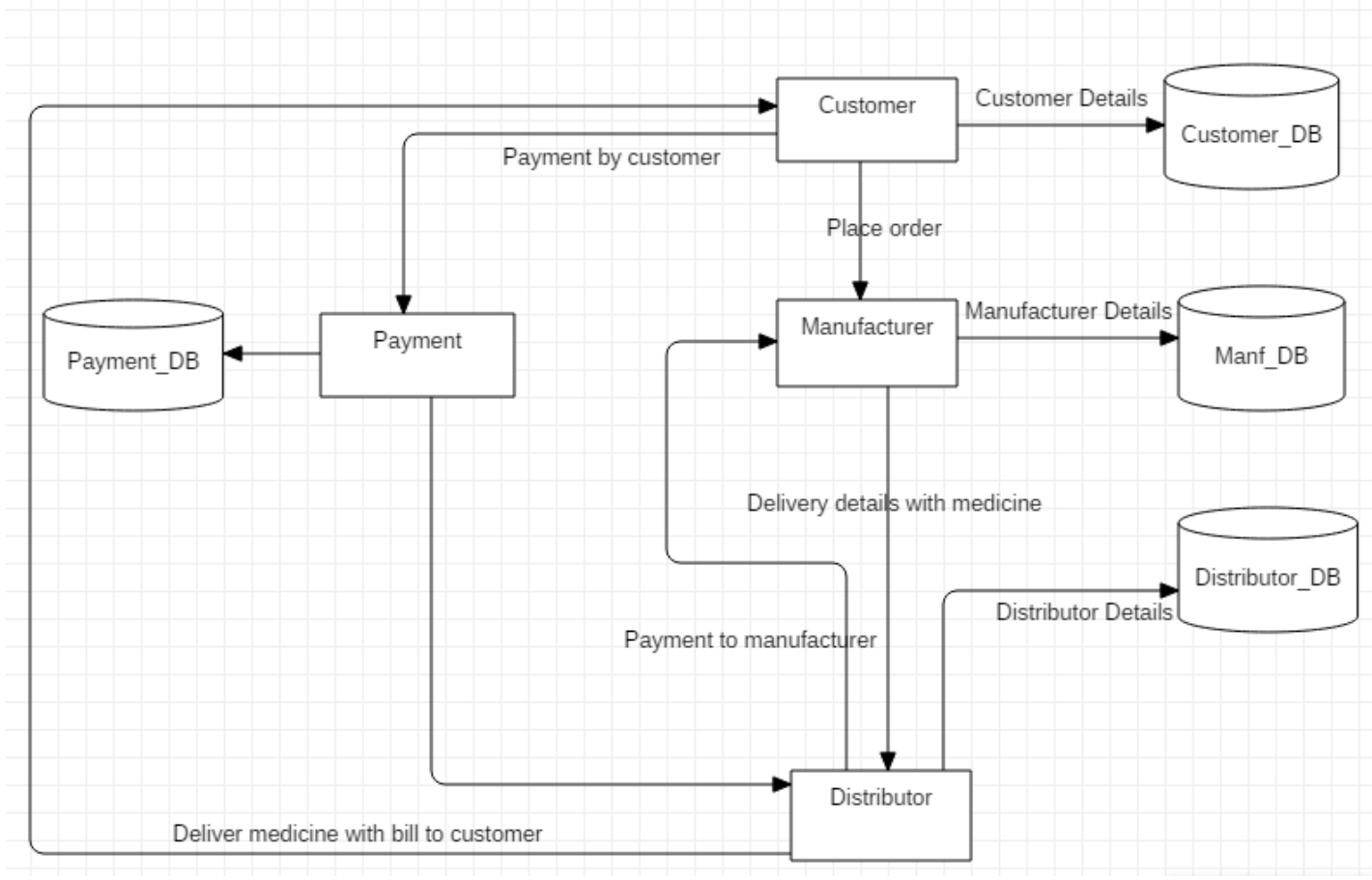

Figure 1 : System Architecture of Management System

\section{IV.MAThematical Model}

\section{Set Theory Model}

Let us consider $\mathrm{S}$ be a System which is denoted by the set of finite elements. $S=\{\mathrm{I}, \mathrm{F}, \mathrm{O}\}$ where,

$\mathrm{I}=$ Input, $\mathrm{F}=$ Function, $\mathrm{O}=$ Output

$\mathrm{F}=\{\mathrm{F} 1, \mathrm{~F} 2, \mathrm{~F} 3\}$

$\mathrm{F} 1=$ Data Block Creation

F2 = Hash Function

F3 = Blockchain Network Creation 
For Data Block Creation,

I1 : $\{\mathrm{CuD} 1, \mathrm{MaD} 1, \mathrm{CaD} 1, \mathrm{CoD} 1\}$

where,

CuD1 = Customer Details

MaD1 = Manufacturer Details

$\mathrm{CaD} 1=$ Carrier Details

CoD1 = Company Details

O1 : F1(I1), Create data block by collecting different details

$\mathrm{O} 1:\{\mathrm{DB} 1 \ldots \mathrm{DBn}\}$ gives different Data blocks

For Hash Function,

I2 : $\{\mathrm{DB} 1 \ldots \mathrm{DBn}\}$

where,

O1 : Data blocks created by F1

$\mathrm{O} 2$ : F2(I2), Calculate hash value for every block

$\mathrm{O} 2:\{\mathrm{H} 1 \ldots \mathrm{Hn}\}$ hash values for every data block

For Blochchain Network Creation,

$\mathrm{I} 3=\{\mathrm{O} 1, \mathrm{O} 2\}$

where,

O1 : Data blocks created by F1

$\mathrm{O} 2$ : Hash values for each block

O3 : F3(I3)

Gives Blockchain Network with composed of different hash values for $\mathrm{n}$ data blocks

\title{
V. Alogorithm
}

Blockchain, Challange is occur to system that to verify blocks are connected to each other or not. For this, some algorithm are used such as Proof of Work, Hash Algorithm.

\section{Proof of Work}

i. Ethash is a PoW of algorithm used in Ethereum.

ii . Ethash aims to provide a PoW algorithm for every commodity hardware for acheive high optimization.

iii. In Blockchain, this algorithm is used to transfer data and produce new blocks to the chain.

iv. The main working principles are a complicated mathematical puzzle and possibility to easily prove the solution for puzzle.

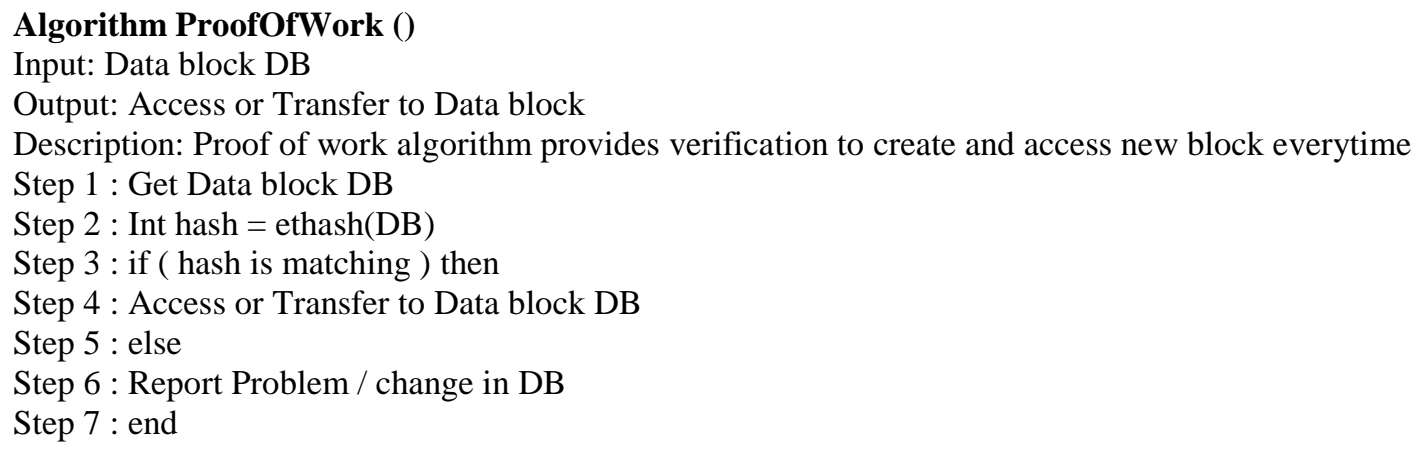

\section{Hashing}

i. To solve the PoW problem or mathematical equation it uses hash.

ii. Hash Algorithm is used to create hash values for each block.

iii. But as network is growing, it is facing more and more difficulties i.e. algorithm needs more hash power to solve.

iv. Accurate work and speed of blockchain system depends on hash values.

\author{
Algorithm ethash (DB) \\ Input: Data block DB \\ Output: Hash Value of Data block
}


Step $1:$ Int Hash=0

Step $2:$ Hash $=$ hash ()

Step 3 : Return Hash

\section{VI.CONCLUSION}

Fierce competition in today's pharmaceutical companies, the introduction of medicines with shorter life cycles and the more expectations of customers have forced business enterprises to invest in, and focus attention on, their supply chains. In typical supply chain, raw materials of medicine are procured and items are produced at one or more pharmaceutical industry shipped to warehouses for intermediate storage, and the shipped to retailers and then shipped to customer.

As this traditional supply chain method is time consuming so, to overcome on this method we are implementing supply chain using blockchain technology to supply medicine from manufacturer to customer in optimal time. This project concludes that blockchain successfully avoid the counterfeit of medicine. This process takes minimum time, money and manpower to transfer medicine. It is the safe way for health consciousness.

\section{ACKNOWLEDGMENT}

We are thankful to our Principal Dr. R. S. Bichkar and HOD Dr. S. K. Shinde for their support in project. At this juncture we feel deeply honored in expressing our sincere thanks to our Guide Mrs. G. J. Chhajed for making the resources available at right time and providing valuable insights leading to successful completion of project.

\section{REFERENCES}

[1]. Blockchains Everywhere - A Use-case of Blockchains in the Pharma Supply-Chain-Thomas Bocek, Bruno B. Rodrigues , Tim Strasser, Burkhard Stiller.

[2]. An Overview on Pharmaceutical Supply Chain: A Next Step towards Good Manufacturing Practice.

[3]. The Pharmaceutical Supply Chain: a Diagnosis of the Stateof- the-Art.

[4]. Block-Supply Chain: A New Anti-Counterfeiting Supply Chain Using NFC and Blockchain

[5]. Blockchains impact on supply chain of a pharmaceutical company

[6]. Pharmaceutical supply chains: key issues and strategies for optimisation

[7]. Pharmaceutical supply strategies

[8].https://www.iotcoresoft.com/iot-knowlegde-center/how-blockchaincan-help-pharmaceutical-supply-chain-management.

[9].https://www.ey.com/Publication/vwLUAssets/ey-blockchain-and-the-supply-chain-three/FILE/ey-blockchain-and-the-supply- chain-three.pdf [10].https://www2.deloitte.com/content/dam/Deloitte/lu/Documents/technology/lu-blockchain-internet-things-supply-chain-traceability.pdf

[11].https://cointelegraph.com/bitcoin-for-beginners/how-blockchain-technology-works-guide-for-beginners-where-can-blockchain-be-used 